\title{
Características de la canal de terneros de la Denominación de Origen Protegida Mirandesa
}

\author{
Sousa, F. ; Lorenzo, J.M. ${ }^{2}$; Iglesias, A. ${ }^{3}$; Cantalapiedra, J. ${ }^{4}$ y Franco, D. ${ }^{2}$
}

'Escola Superior Agrária. Instituto Politécnico de Bragança. Portugal.

${ }^{2}$ Centro Tecnológico de la Carne de Galicia. Ourense. Spain.

${ }^{3}$ Departamento de Anatomía y Producción Animal. Universidad de Santiago de Compostela. Lugo. Spain.

${ }^{4}$ Farm Counselling Services. Consellería do Medio Rural. Xunta de Galicia. Lugo. Spain.

\section{PaLABRAS CLAVE ADICIONALES}

Raza autóctona.

Despiece de la canal.

Quinto cuarto.
Medidas morfométricas.

\section{RESUMO}

El objetivo de este trabajo fue evaluar las características de la canal de la raza Mirandesa. Para llevar a cabo este estudio, un total de 20 animales de la raza Mirandesa fueron sacrificados a los 315 días. 24 horas tras el sacrificio, se determinó el peso canal y rendimiento canal, se realizaron las medidas morfométricas (longitud de la canal, longitud de la pierna, espesor de la pierna, profundidad interna del pecho y perímetro máximo de la pierna), se calcularon los siguientes índices: índice de compacidad de la canal e índice longitudinal transversal de la pierna y se realizó el despiece de los principales cortes comerciales. Los animales de la raza Mirandesa presentaron un peso vivo de $293,7 \mathrm{~kg}$, un peso canal de $152 \mathrm{~kg}$ y un rendimiento canal de $60,5 \%$. En relación a las medidas morfométricas, la raza Mirandesa mostró una longitud de canal de $110 \mathrm{~cm}$, una longitud de pierna de $69 \mathrm{~cm}$ y un perímetro de pierna de $89,8 \mathrm{~cm}$. Los valores para el índice de compacidad de la canal y el índice longitudinal transversal de la pierna fueron de 1,4 y 3,7 , respectivamente. Con respecto al quinto cuarto, la piel presentó los mayores porcentajes seguida de la cabeza y pies. Finalmente, la composición de las canales de la raza Mirandesa fue: $74,9 \%$ de carne, $18,1 \%$ de hueso y $7,0 \%$ de grasa.

\section{Carcass characteristic of calves from Protected Designation of Origin Mirandesa breed}

\section{SUMMARY}

\section{ADDITIONAL KEYWORDS}

Autochthonous breed.

Morphometric measurements.

Quartering of the carcass.

Fifth quarter.

INFORMACIÓN

\section{Cronología del artículo.}

Recibido/Received: 02.12.2015

Aceptado/Accepted: 14.03.2016

On-line: 11.06 .2016

Correspondencia a los autores/Contact e-mail:

imlorenzo@ceteca.net

\section{INTRODUCCIÓN}

En los últimos 15 años el uso de la DOP (Denominación de Origen Protegida) ha constituido el mejor instrumento de conservación de las razas bovinas en peligro de extinción, así como de sus sistemas de producción. En el año 2007, la carne de bovino co-
The aim of this study was to evaluate the carcass characteristics of Mirandesa breed. To carry out this study, a total of 20 animals of Mirandesa breed were slaughter at 315 days. After 24 hours of slaughter, carcass weight and dressing percentage were determined, and morphometric measurements (carcass length, leg length, leg width, leg perimeter, chest external depth, chest internal depth and leg maximum perimeter) were performed and the following indexes were calculated: carcass compactness index and hindlimb compactness index and the main commercial cuts were obtained. Animals from Mirandesa breed presented a $293.7 \mathrm{~kg}$ live weight, a $152 \mathrm{~kg}$ carcass weight and a dressing percentage of $60.5 \%$. Regarding morphometric measurements, animals from Mirandesa breed showed a carcass length of $110 \mathrm{~cm}$, leg length of $69 \mathrm{~cm}$ and leg perimeter of $89.8 \mathrm{~cm}$. The values for the carcass compactness index and hindlimb compactness index were 1.4 and 3.7, respectively. Respecting fifth quarter, skin showed the highest percentages following by head and feet. Finally, the percentages of Mirandesa breed's carcass quartering were $74.9 \%$ for meat, $18.1 \%$ for bone and $7.0 \%$ for fat. mercializada bajo el sello de las 9 DOP que existían en Portugal suponía el 2,3\% respecto del total producido, pero mientras la producción de carne de bovino a nivel europeo se ha ralentizado desde el año 2011 (FAOSTAT, 2013), la producción de carne con DOP tiene una aceptación mayor porque las preferencias de los consumidores por productos naturales, producidos 
bajo sistemas tradicionales y respetuosos con el medio ambiente han crecido (Hermansen, 2003). Además, la calidad de los productos que aporta la raza bovina Mirandesa esta contrastada, como en el caso de la Posta Mirandesa, un plato muy apreciado dentro de la gastronomía portuguesa, y por el hecho de que dentro del ranking de carne de bovino con DOP en Portugal es la que alcanza los precios más altos para los productores $(5,25 € / \mathrm{kg})$ (Sousa et al., 2012).

En la bibliografía se pueden encontrar numerosos trabajos relacionados con los rendimientos productivos, la calidad de la canal y de la carne de distintas razas autóctonas (Albertí et al., 2001, 2005, 2008; Piedrafita et al., 2003) pero apenas se dispone de información de la raza Mirandesa. Esta raza es originaria del noreste de Portugal (Trás-os-Montes), cerca de la frontera española. En la década de los 80, la raza Mirandesa sufrió un enorme descenso de sus efectivos, siendo clasificada en peligro de extinción en al año 1994. Posteriormente, en el año 1995, se aprobó la Denominación de Origen Protegida de la Carne de Mirandesa, situándose en el año 2007 como la segunda dentro de las carnes portuguesas con DOP, con un volumen del 13\% y 254 tn (Sousa y Sánchez, 2009). En aquel momento, la Asociación Profesional de la Raza Mirandesa la constituían 630 ganaderos y un rebaño de 6000 vacas. Actualmente el sistema de producción se basa en pequeñas explotaciones familiares que emplean sistemas tradicionales basados en la alimentación con leche materna suplementada con pienso, maíz, patatas, silo de maíz y forrajes producidos en la explotación familiar o de pastoreo donde los animales son finalizados con concentrado (2-4 meses). La venta de los terneros se lleva a cabo a edades comprendidas entre 5 y 8 meses cuando los animales son destetados (Fragata y Sousa, 1997).

Hoy en día, la creciente preocupación por encontrar formas de producción sustentables y en equilibrio con el medio ambiente, sumado a la preservación de la cultura tradicional y el patrimonio histórico, han dado sentido para que se tomen las medidas oportunas para que esta raza bovina siga existiendo. Para preservar este patrimonio es necesario profundizar en el estudio de las características que hacen de ella una raza de interés económico. En base a lo expuesto anteriormente, el objetivo de este trabajo fue evaluar la calidad de la canal (medidas morfométricas, despiece y composición del quinto cuarto) de terneros machos de raza Mirandesa sacrificados como posible producto distinguible mediante una marca de calidad, orientada hacia el turismo y restauración.

\section{MATERIAL Y MÉTODOS}

Se emplearon 20 animales machos enteros de la raza Mirandesa (inscritos en el libro genealógico de la raza Mirandesa) con una edad al sacrificio aproximada de 10 meses y medio. Los animales fueron criados en un sistema de producción extensivo, donde los animales, después del destete, fueron sometidos a un periodo de finalización de 120 días en el puesto Zootécnico de Malhadas, bajo el control de técnicos de la Denominación de Origen Mirandesa. Todos los animales fueron pesados empleando una báscula electrónica (TRU TEST) con capacidad hasta $1000 \mathrm{~kg}$, precisión de 100 g y un error del $1 \%$, obteniéndose el peso vivo (PV) antes del sacrifico. Durante el periodo de acabado fueron alimentados con heno de pradera ad libitum, avena y una mezcla de harinas; la mezcla tenía la siguiente formulación: $70 \mathrm{~kg}$ centeno, $440 \mathrm{~kg}$ de cebada, $200 \mathrm{~kg}$ de maíz, $210 \mathrm{~kg}$ de bagazo de soja, $30 \mathrm{~kg}$ de melaza y núcleo de $50 \mathrm{~kg}$ (vitamina A, D, E, minerales correctivos y aceite de palma hidrogenado). Desde el día anterior al sacrificio los animales estuvieron en ayunas, siendo transportados desde Malhadas (Portugal) a las 8 de la mañana hasta un matadero acreditado en Miranda de Douro (Portugal) situado a 8 kilómetros. Todos los animales fueron sacrificados antes de las 10 de la mañana para minimizar posibles problemas de estrés pre-sacrificio. Se obtuvo el peso de la canal caliente (PCC) a los 45 minutos y el peso canal fría (PCF) después de un periodo de refrigeración de $24 \mathrm{~h}$ a $2{ }^{\circ} \mathrm{C}$. A continuación se tomaron las medidas morfométricas en la canal propuestas por De Boer et al. (1974) y Carballo et al. (2000): longitud de la canal (LC), longitud de la pierna (LP) espesor de la pierna (EP), profundidad interna del pecho (PIP) y perímetro máximo de la pierna (PL). Teniendo en cuenta los parámetros anteriores se calcularon los siguientes índices (Espejo et al., 2000): índice de compacidad de la canal $(\mathrm{ICC})=(\mathrm{PCF} / \mathrm{LC})$ e índice longitudinal transversal de la pierna $($ ILTP $)=($ LP $/ E P)$. El peso vivo vacío (PVV) fue calculado como: PV día del sacrificio -[Peso reservorios gástricos llenos (kg-Pesos reservorios gástricos vacíos $(\mathrm{kg})$ - Peso intestinos llenos (kg)- Peso intestinos vacíos (kg)]. El rendimiento canal verdadero (RCC) y las perdidas por oreo (PO), expresadas en porcentaje, se calcularon de acuerdo a las siguientes ecuaciones: $\mathrm{RCC}=(\mathrm{PCF} / \mathrm{PV}) \times 100$ y $\mathrm{PF}=$ [1- (PCF/PCC)] x 100, respectivamente. Posteriormente, se procedió al despiece de la media canal izquierda, anotándose el peso de cada pieza comercial y calculando el porcentaje que representaba respecto a la canal, agrupándose posteriormente según los tipos de tejidos y categorías de las piezas comerciales. La metodología empleada en el despiece es la descrita por Soares (1959). Para la determinación de la composición tisular, las piezas fueron preparadas de acuerdo con las buenas prácticas comerciales, manteniéndose la cobertura de grasa intermuscular y de tejido conectivo normal. Los huesos fueron limpiados de acuerdo con las prácticas comerciales, manteniendo algunos fascias, eliminado la grasa perirrenal. Todo ello con el objetivo de estimar, en términos porcentuales, la porción de carne, hueso y grasa comerciales que comprende la canal. De acuerdo con la metodología indicada por Carballo et al. (2000), en la porción de lomo extraído a las 48 horas post-mortem y sobre papel de acetato y con un rotulador de punta fina, se dibujó el perímetro del músculo L. thoracis de las costillas $6^{\mathrm{a}}$ y $10^{\mathrm{a}}$. Posteriormente, se determinó su superficie con un planímetro digital Koisumi KP-90 Placom, dando los resultados en $\mathrm{cm}^{2}$. El análisis estadístico de los resultados se realizó mediante el programa estadístico SPSS (versión 19.0) realizando previamente un análisis de los datos mediante diagrama de cajas para comprobar la existencia de valores anómalos.

\section{RESULTADOS Y DISCUSIÓN}

La tabla I muestra la media, desviación estándar, máximo y mínimo de las características de la canal de machos de la raza Mirandesa. Los valores de peso del 
animal vivo y canal fría oscilaron entre 231-393 kg y $116-208 \mathrm{~kg}$, respectivamente. Estos resultados fueron similares a los observados por Aveiro (1998) y Ferreira (1998) para animales de la misma raza sacrificados a los 260 días. Franco et al. (2010) también observaron valores similares para terneros de la raza Limiá (301 y $136 \mathrm{~kg}$ para los pesos vivo y canal, respectivamente) sacrificados entre 7 y 11 meses y para terneros de la raza Vianesa (273 y $129 \mathrm{~kg}$ para los pesos vivo y canal, respectivamente) sacrificados a 9 meses de edad. Sin embargo, estos resultados fueron inferiores a los encontrados por Carballo et al. (2004) quienes observaron valores de 323 y $185 \mathrm{~kg}$ para el peso vivo y el peso canal, respectivamente, en animales de la raza Rubia Gallega con edades comprendidas entre los 8 y 10 meses de edad. Estas diferencias pueden ser debidas a la diferente aptitud cárnica entre ambas razas y al sistema de acabado. El rendimiento canal en matadero mostró un valor medio del 60,5\% con máximos, en algunos casos, superiores al 64\%, lo cual es una cifra muy notable. Estos rendimientos canal fueron superiores a los encontrados por Simões et al. (2005) en razas Portuguesas de menor tamaño como la Arouquesa (53\%), Barrosa (54\%), Maronesa (55\%) y Mertolenga (56\%) sacrificados con un peso vivo de $300 \mathrm{~kg}$; y para razas de mayor formato como la Alentejana (55\%), Mirandesa (58\%) y la Marinhoa (58\%) sacrificados a $400 \mathrm{~kg}$. Para alcanzar rendimientos canal superiores al $60 \%$ es necesario, en las razas indicadas, sacrificar a mayor peso vivo, como en el caso de animales de la raza Mirandesa y de Marinhoa sacrificados con $650 \mathrm{~kg}$, que alcanzaron rendimientos canal del 62 y $63 \%$, respectivamente. Los rendimientos canal en el presente trabajo también fueron superiores a los obtenidos por Carballo et al. (2004) en Rubia Gallega (57,3\%), Arthur et al. (1995) para Angus (55,7\%), Hereford (56,6\%) y Charolesa (55,6\%) y por Piedrafita et al. (2003) para Asturiana de la Montaña (56,3\%), Avileña Negra Ibérica (58,1\%), Morucha (57,2\%), Retinta (57,5\%), Aubrac (59,9\%) y Salers $(58,5 \%)$. Estas diferencias en el rendimiento canal entre razas podrían ser debidas a las diferentes edades de sacrificio a las que fueron sacrificados los animales, sistema de producción y tasas de crecimiento inherentes a cada raza.

Cuando se sacrifica un animal, además de la canal se obtiene un conjunto de productos y vísceras a los que, en el pasado, los productores daban poca importancia, porque habitualmente no se pagaban. Sin embargo, hoy en día la situación ha cambiado y ha aumentado la necesidad de avanzar en la cadena de valor para seguir siendo competitivos, por lo que las vísceras y subproductos tienen un valor comercial cada vez más significativo. En la tabla II se muestran los valores porcentuales, respecto al peso vivo de los principales subproductos y vísceras de los terneros de raza Mirandesa. La piel es el subproducto de mayor importancia $(11,2 \%)$, lo que también coincide por ser el de mayor valorización e interés comercial que despierta en el productor. Los valores medios porcentuales de cabeza $(4,8 \%)$ del presente trabajo fueron muy superiores a los mostrados por Simões et al. (2005) en diferente razas Portuguesas con valores extremos de $2,4 \%$ para Alentejana y de 2,8\% para Mertolenga. Lo mismo ocurre con el porcentaje de pies (2,9\%) superior también en los animales de este trabajo en comparación con los obtenidos por Simões et al. (2005) (1,8-2,2\%). Cuando se compara el tracto digestivo, suma de estómagos e intestinos, los valores medios obtenidos para la raza Mirandesa (3,6\%) fueron inferiores a los descritos por Simões et al. (2005) quienes observaron valores entre $4,1 \%$ y 5,4\% para la raza Arouquesa y Barrosa, respectivamente. Con respecto al porcentaje de vísceras, calculado como la suma de pulmón, corazón, hígado, bazo y riñones, nuestros resultados (4,2\%) fueron ligeramente superiores a los observados por Simões et al. (2005) para la raza Mirandesa (3,8\%). Por último, destacar que las comparaciones deben hacerse con cautela debido a las diferencias de edad lo que genera diferencias alométricas en los diferentes tejidos entre los animales, además de ligeras diferencias metodológicas que pudieran darse.

En la tabla III se presentan los valores medios, desviación estándar, máximo y mínimo de las medidas objetivas de la canal. Los valores medios de la longitud oscilaron entre 104 y $120 \mathrm{~cm}$, con un valor medio de $110,1 \mathrm{~cm}$. Estos resultados coinciden los publicados previamente por Albertí et al. (2005) quienes mostraron valores medios de longitud de canal de 109,8 cm en siete razas españolas y por Franco et al. (2010) para terneros de las raza Vianesa y Limiá $(108,5 \mathrm{~cm})$. Sin embargo, Albertí et al. (2008) encontraron valores de longitud de canal ligeramente superiores en animales con un peso canal similar al nuestro: Casina $(121,1 \mathrm{~cm})$, Highland $(119,1 \mathrm{~cm})$ y Jersey $(119,8 \mathrm{~cm})$ y Sánchez et al. (2005) para Nelore $(126 \mathrm{~cm})$. Respecto a las variables relacionadas con el volumen de la pierna como el perímetro y espesor, también fueron ligeramente superiores en los terneros de este trabajo que en razas Gallegas (Franco et al., 2010).

Tabla I. Media, desviación estándar, máximo y mínimo de las características de la canal de machos de la raza Mirandesa $(n=20)$ (Mean, standard deviation, maximum and minimum of carcass characteristics from male Mirandesa breed $(n=20)$.

\begin{tabular}{lllll}
\hline & Media & SD & MAX & MIN \\
\hline Edad (días) & 312,80 & 23,30 & 364,00 & 280,00 \\
Peso vivo (kg) & 293,65 & 46,98 & 393,00 & 231,00 \\
Peso vivo vacío (kg) & 250,53 & 39,94 & 333,20 & 200,90 \\
Peso canal caliente(kg) & 154,94 & 28,61 & 212,10 & 118,20 \\
Peso canal fría (kg) & 151,99 & 27,80 & 208,40 & 116,80 \\
Rendimiento canal verdadero (\%) & 60,48 & 1,72 & 64,10 & 57,49 \\
Pérdidas por oreo (\%) & 1,87 & 0,83 & 3,55 & 0,61 \\
\hline
\end{tabular}


Tabla II. Media, desviación estándar máximo y mínimo de los componentes del porcentaje del quinto cuarto (respecto al peso vivo vacío) de machos de la raza Mirandesa $(n=20)$ (Mean, standard deviation, maximum and minimum of the percentage of fifth quarter components (relative to live weight empty) from male Mirandesa breed $(n=20)$.

\begin{tabular}{lllll}
\hline & Media & SD & MAX & MIN \\
\hline Peso canal fría $(\mathrm{kg})$ & 151,99 & 27,80 & 208,40 & 116,80 \\
Piel & 11,19 & 0,53 & 12,44 & 9,94 \\
Intestinos* $^{*}$ & 1,73 & 0,37 & 2,36 & 0,78 \\
Cabeza $^{*}$ & 4,83 & 0,16 & 5,11 & 4,58 \\
Estómagos $^{*}$ & 1,90 & 0,29 & 2,26 & 0,90 \\
Pies & 2,90 & 0,17 & 3,23 & 2,60 \\
Hígado & 1,45 & 0,15 & 1,70 & 1,14 \\
Pulmón & 1,66 & 0,33 & 2,28 & 1,19 \\
Corazón & 0,43 & 0,06 & 0,54 & 0,31 \\
Lengua & 0,41 & 0,07 & 0,54 & 0,30 \\
Rabo & 0,39 & 0,04 & 0,50 & 0,27 \\
Riñón & 0,29 & 0,05 & 0,40 & 0,18 \\
Bazo & 0,36 & 0,07 & 0,49 & 0,26 \\
\hline *Intestinos y estómagos sin su contenido, & &
\end{tabular}

El índice de compacidad de la canal, que denota la compacidad de la misma y que se muestra como un buen discriminador para los distintos grados de conformación al ser mayor en las mejores categorías, presentó un valor medio de 1,38. Los valores para el índice de compacidad de la canal de los terneros de las razas Limiá y Vianesa fueron cercanos y de media 1,25 y 1,17 respectivamente, para animales de conformación media O. En línea con esto, Carballo et al. (2004) observaron que el índice de compacidad de la canal aumentaba a medida que mejoraba la conformación, pasando de 1,38 a 1,95 en terneros de raza Rubia Gallega de conformaciones $\mathrm{O}$ y E, respectivamente. Con respecto al índice longitudinal transverso de la pierna, nuestros valores medios fueron de $3,70(4,25-3,36)$, mejores que los mostrados por Franco et al. (2010) para terneros de las razas Limiá y Vianesa con valores de 4,31 y 4,51, respectivamente; pero inferiores a los obtenidos por Carballo et al. (2004) en terneros de raza Rubia Gallega con valores de 3,11 para terneros de conformación $\mathrm{R}$ y $\mathrm{O}$ y con valores de 2,67 y 2,77 para conformaciones E y U, respectivamente. Es este sentido, Varela (2002) afirmó que las diferencias en el ILTP son consecuencia lógica de las diferencias en el espesor de la pierna y que pueden ser consecuencia de una mejor conformación de la raza.

Un método indirecto para valorar la calidad de la canal radica en las medidas de área de musculo Longissimus thoracis a diferentes niveles (sexta y décima costilla, véase tabla III), información que además se puede emplear para determinar el desarrollo de la masa muscular. En nuestro estudio obtuvimos valores medios de 31,3 y $57,5 \mathrm{~cm}^{2}$ para la sexta y décima costilla, respectivamente. Según los trabajos llevados a cabo por Carballo et al. (2004) estás área se corresponderían con una categoría comercial O. Estos mismos autores han señalado que el área de la décima costilla ofrecía mejores resultados en términos de clasificación de canales que la sexta costilla. Sin embargo, en nuestro estudio no se pudo comprobar dicha afirmación ya que no dispusimos de la conformación de las canales.

La tabla IV muestra los valores medios, desviación estándar, máximo y mínimo de los valores obtenidos del despiece de la media canal izquierda, expresado en porcentaje, así como la composición tisular. La canal es la principal unidad de transacción entre los sectores productivos y comercializador de carne de bovino, por lo que es fundamental conocer su composición y rendimiento de las diferentes partes que la integran para poder así estimar su verdadero valor comercial. La variabilidad de sus componentes físicos y su distinta distribución en la canal da lugar a que las piezas

Tabla III. Media, desviación estándar, máximo y mínimo de las medidas morfométricas de la canal de machos de la raza Mirandesa $(n=20)$ (Mean, standard deviation, maximum and minimum of morphometric measurements from male Mirandesa breed $(n=20)$.

\begin{tabular}{|c|c|c|c|c|}
\hline & Media & SD & Max & Min \\
\hline Peso canal fría (kg) & 151,99 & 27,80 & 208,40 & 116,80 \\
\hline \multicolumn{5}{|l|}{ Medidas morfométricas $(\mathrm{cm})$} \\
\hline Longitud canal & 110,10 & 4,45 & 120,00 & 104,00 \\
\hline Longitud pierna & 69,03 & 2,21 & 75,00 & 66,00 \\
\hline Perímetro pierna & 89,78 & 4,15 & 97,50 & 82,00 \\
\hline Profundidad interna pecho & 28,65 & 1,18 & 31,00 & 26,00 \\
\hline Espesor pierna & 18,70 & 1,27 & 21,50 & 16,00 \\
\hline \multicolumn{5}{|l|}{ Índices de la canal } \\
\hline ICC & 1,38 & 0,20 & 1,75 & 1,09 \\
\hline ILPT & 3,70 & 0,19 & 4,25 & 3,36 \\
\hline \multicolumn{5}{|c|}{ Área del m. Longissimus thoracis $\left(\mathrm{cm}^{2}\right)$} \\
\hline $6^{\text {a }}$ costilla & 31,28 & 5,16 & 42,89 & 22,32 \\
\hline $10^{\mathrm{a}}$ costilla & 57,49 & 9,71 & 79,92 & 43,93 \\
\hline
\end{tabular}


Tabla IV. Media, desviación estándar, máximo y mínimo del despiece de la canal (\%) de machos de la raza Mirandesa $(n=20)$ (Mean, standard deviation, maximum and minimum of the quartering of the carcass $(\%)$ from male Mirandesa breed $(n=20)$.

\begin{tabular}{|c|c|c|c|c|}
\hline & Media & SD & Max & Min \\
\hline Peso canal fría $(\mathrm{kg})$ & 151,99 & 27,80 & 208,40 & 116,80 \\
\hline Lomo & 7,47 & 0,55 & 8,70 & 6,56 \\
\hline Solomillo & 2,24 & 0,2 & 2,70 & 1,82 \\
\hline Cadera & 3,89 & 0,36 & 4,45 & 3,04 \\
\hline Tapa & 7,06 & 0,53 & 8,43 & 6,29 \\
\hline Babilla & 4,12 & 0,37 & 4,86 & 3,53 \\
\hline Contra & 5,08 & 0,37 & 5,93 & 4,50 \\
\hline Redondo & 1,80 & 0,21 & 2,31 & 1,50 \\
\hline Jarrete & 3,66 & 0,41 & 5,05 & 3,24 \\
\hline Espalda & 7,84 & 1,15 & 12,32 & 7,01 \\
\hline Aguja+Pescuezo & 10,34 & 0,91 & 12,17 & 8,43 \\
\hline Pecho & 5,24 & 0,92 & 6,58 & 3,73 \\
\hline Jarrete & 3,25 & 0,38 & 3,84 & 2,47 \\
\hline \multicolumn{5}{|c|}{ Composición tisular (\%) } \\
\hline Carne & 74,85 & 2,24 & 78,32 & 71,39 \\
\hline Hueso & 18,12 & 0,98 & 19,81 & 15,65 \\
\hline Grasa & 7,03 & 1,75 & 9,33 & 4,12 \\
\hline Carne/hueso & 4,15 & 0,33 & 5,00 & 3,70 \\
\hline
\end{tabular}

que se obtienen de ella presenten distintas aptitudes culinarias, lo que se traduce en un precio comercial diferenciado. Los valores medios obtenidos del despiece de las canales de la raza Mirandesa fueron de $74,9 \%$ para carne, $18,1 \%$ para hueso y $7,0 \%$ para grasa. Esta proporción de carne se encuentra en la parte superior del intervalo de valores $(70-76 \%)$ observado por otros autores (Piedrafita et al., 2003; Albertí et al., 2008; Franco et al., 2010) para razas autóctonas españolas (Cachena, Caldelá, Limiá, Vianesa, Asturiana de los Valles, Morucha, Pirenaica y Retinta). Para terneros de la raza Rubia Gallega de conformación alta (U y E) se llegan a rendimientos de carne de $78,4 \%$ y $80,5 \%$, respectivamente; mientras que conformaciones inferiores ( $\mathrm{R} \mathrm{y} \mathrm{O}$ ) no superan el 75\% (Carballo et al., 2004). Sin embargo, Simões y Mendes (2003) obtuvieron porcentajes de carne en la canal del 67,8\% para la raza Mirandesa. Con respecto a los porcentajes de hueso y grasa, nuestros valores medios están dentro del intervalo encontrado por Albertí et al. (2001) quienes obtuvieron porcentajes de $16-19 \%$ y $7-10 \%$ para hueso y de grasa, respectivamente. Por el contario, Simões y Mendes (2003) encontraron porcentajes ligeramente inferiores de hueso $(15,4-17,4 \%)$ y del doble para la grasa $(13,1-16,0 \%)$ en la raza Mirandesa.

Por otro lado, el cuarto trasero de la canal que representa aproximadamente $2 / 3$ de la carne vendible, y es donde se encuentran las piezas de mayor valor comercial, tanto de categoría extra (lomo y solomillo) como piezas de primera (cadera, tapa, babilla, contra y redondo), representaron un $9,7 \%$ y $21,9 \%$ del peso de la canal, respectivamente. Respecto a las piezas de segunda (espalda, aguja y pescuezo) y las piezas de tercera (jarretes delantero y trasero y el pecho), se obtuvieron porcentajes respecto de la canal del 18,2\% y
$12,2 \%$, respectivamente. Por último, la relación carne/ hueso fue similar a la mostrada por terneros de raza Rubia Gallega de conformación R $(4,09)$ (Carballo et al., 2004), superior a la mostrada por razas autóctonas Gallegas (Franco et al., 2010) con valores no superiores a 3,21 e inferior a la presentada por terneros de raza Rubia Gallega de conformación alta (U y E con valores de 4,97 y 5,43, respectivamente) (Carballo et al., 2004).

\section{CONCLUSIONES}

No existen demasiados datos en la bibliografía relativos a la calidad de canal de la raza Mirandesa, siendo esta una de las razas autóctonas de Portugal de mayor formato junto con la Alentejana y Marinhoa, por lo que los resultados que aquí se presentan sirven para caracterizar mejor esta raza. A la luz de los mismos, podemos concluir que estamos ante una raza que presenta unos notables rendimiento canal en algunos individuos con valores superiores al $64 \%$, teniendo en cuenta la rusticidad de la raza y los pesos de sacrificio a los que se consigue dicho rendimiento. Asimismo, el despiece nos permitió comprobar que estamos ante una raza que proporciona tenernos con un alto porcentaje de carne (valores próximos al 75\%) lo cual es de sumo interés para la industria a la hora de fomentar esta raza, y con relaciones carne/hueso equivalentes a las mostradas en terneros de razas de aptitud cárnica como la Rubia Gallega.

\section{BIBLIOGRAFÍA}

Albertí, P.; Lahoz, F.; Tena, R.; Jaime, S.; Sañudo, C.; Olleta, J.L.; Campo, M.M.; Panea, B. y Pardos, J.J. 2001. Producción y rendimiento carnicero de siete razas bovinas españolas faenadas a distintos pesos. 
Dirección General de Tecnología Agraria. Zaragoza. Informaciones Técnicas, 101: 1-16.

Albertí, P.; Panea, B.; Sañudo, C.; Olleta, J. L.; Ripoll, G.; Ertbjerg, P. and Williams, J.L. 2008. Live weight, body size and carcass characteristics of young bulls of fifteen European breeds. Livest Sci, 114: 19-30.

Albertí, P.; Ripoll, G.; Goyache, F.; Lahoz, F.; Olleta, J.L.; Panea, B. and Sañudo, C. 2005. Carcass characterisation of seven Spanish beefbreeds slaughtered at two commercial weights. Meat Sci, 71: 514-521.

Arthur, P.D.; Hearnshaw, H.; Jonhoston, D. and Stephenson, P.D. 1995. Evaluation of Angus, Charolais and Hereford as terminal sire breeds on Hereford and first-cross cows. II.- Carcasses characteristics and retail yield of progeny. Aust J Agric Res, 46: 1245-1258.

Aveiro, D.M.M. 1998. Os bovinos de raça Mirandesa, avaliação dos resultados dos testes de performance em bezerros nos anos de 1997/1998. Curso de Produção Animal. Instituto Politécnico de Bragança.

Carballo, J.A.; Monserrat, L. y Sánchez, L. 2000. Composición de la canal bovina. En: Cañeque, V. y Sañudo, C. (Eds.) Metodología para el estudio de la calidad de la canal y de la carne en rumiantes. Instituto Nacional Investigación y Tecnología Agraría y Alimentaria. Madrid. Monografía INIA: Serie Ganadera, 1: 107-123.

Carballo, J.A.; Oliete, B.; Moreno, T.; Sánchez, L. y Monserrat, L. 2004. Categorización de las canales de ternero producidas en Galicia. Arch Zootec, 53: 119-128.

De Boer, H.; Dumont, B.L.; Pomeroy, R.W. and Weniger, J.H. 1974. Manual on E.A.P.P. reference methods for the assessment of carcass characteristics in cattle. Livest Prod Sci, 1: 151-154.

Espejo, M.; García, S.; López, M.M.; Izquierdo, M.; Robles, A. y Costela, A. 2000. Morfología de la canal bovina. En: Cañeque, V. y Sañudo, C. (Eds.). Metodología para el estudio de la calidad de la canal y de la carne. Instituto Nacional Investigación y Tecnología Agraría y Alimentaria. Madrid. Monografía INIA: Serie Ganadera, 1: 69-80.

FAOSTAT. 2013. Agriculture, forestry and fishery statistics. In: Coyette, C. and Schenk, H. (Ed.). Publications Office of the European Union. Luxembourg. $256 \mathrm{pp}$.

Ferreira, M.J.M. 1998. Os bovinos de raca Mirandesa: contribuicao para o estudo do crescimento entre o nascimento e o desmame - Evolucao do peso vivo. Relatorio final de estagio do bacharelato em Producao Animal. Escola Superior Agraria de Braganca.

Fragata A. and Sousa F. 1997. Mirandesa cattle breed: Farm structure, management and marketing. In: J.T. Sorensen (Ed.). Livestock farmin systems. More than food production. Wageningen Pers. Wageningen. pp. 212-216.

Franco, D.; Fernández, M.; Justo, J.R.; Lorenzo, J.M.; González, L.; Rivero, C.J.; Lama, J.; García-Fontán, M.C.; Bispo, E.; Carracedo, S. and Moreno, T. 2010. Meat characterization of four endangered Galician cattle breeds. 56th International Congress of Meat Science and Technology. 15-20 Agosto. Jeju. Korea.

Hermansen, J. E. 2003. Organic livestock production systems and appropriate development in relation to public expectations. Livest Prod Sci, 80: 3-15.

Piedrafita, J.; Quintanilla, R.; Sañudo, C.; Olleta, J. L.; Campo, M. M. and Panea, B. 2003. Carcass quality of 10 beef cattle breeds of the Southwest of Europe in their typical production system. Livest Prod Sci, 82: 1-13.

Sánchez, L.; Carballo, J. A.; Sánchez, B. y Montserrat, L. 2005. Características de la canal y de la carne de machos procedentes del cruce de Rubia Gallega con Nelore. Arch Zootec, 54: 485-489.

Simões, J. and Mendes, I. 2003. Distribution of tissues in carcasses at the same proportion of total fat in Portuguese cattle breeds. Anim Res, 52: 287-298.

Simões, J.A.; Mira, J.F.F.; Lemos, J.P.C. and Mendes, I.A. 2005. Dressing percentage and its relationship with some components of the fifth quarter in Portuguese cattle breeds. Livest Prod Sci, 96: 157-163.

Soares, I. 1959. Definição anatômica das pecas de talho (cortes de Lisboa e do Porto). Junta Nacional dos Produtos Pecuários.

Sousa, F.; Aguiar, C. and Aveiro, D. 2012. Mirandesa meat PDO: The strategies of family farm systems for their governance. International Farming Systems Association (IFSA). 1-4 July 2012. Aarhus. Denmark. Sousa, F.y Sánchez, L. 2009. Mirandesa. Ed. Asociación de los Criadores de Bovinos de Raza Mirandesa. pp. 1-341.

Varela, A. 2002. Estudio de las variables que afectan a la producción del tipo cebón. Tesis doctoral. Universidad de Santiago de Compostela. 